\title{
SATA (Sistema de Acompanhamento do Tratamento de Autismo)
}

\author{
Fernando C.B.G. Santana ${ }^{1}$, Lucas R. de Carvalho ${ }^{1}$, Guilherme N. de Sá Ribeiro ${ }^{1}$, \\ Sandra Elisa Veloso Aguiar ${ }^{1}$, Ana Caroline Rodrigues Santana ${ }^{1,2}$ \\ ${ }^{1}$ Instituto Federal de Educação, Ciência e Tecnologia do Piauí (IFPI) \\ Av. Presidente Jânio Quadros, 330, 64053-390, Santa Isabel - Teresina - Piauí - Brasil \\ ${ }^{2}$ Colégio LEROTE \\ R. Professor Elias Torres, 1020, 64052-160, São Cristóvão - Teresina - Piauí - Brasil \\ fernandosantana@ifpi.edu.br
}

\begin{abstract}
The SATA (Autism Treatment Monitoring System) is a web tool developed to monitor the clinical evolution of patients during the treatment of ASD (Autistic Spectrum Disorder). For the acquisition of carrier data, SATA uses the Childhood Autism Rating Scale (CARS), which is one of the most widely used mechanisms to aid diagnosis and follow-up of the evolution of autism carriers. The results of the evaluations carried out in a particular bearer can be used to create various types of reports on the evolution of the same, facilitating in this way, the choice of new therapies that can boost their gains.
\end{abstract}

Resumo. O SATA (Sistema de Acompanhamento do Tratamento de Autismo) é uma ferramenta web desenvolvida para acompanhar a evolução do quadro clínico de pacientes durante o tratamento do TEA (Transtorno do Espectro Autista). Como padrão para aquisição de dados do portador, o SATA usa a CARS (Childhood Autism Rating Scale) que é um dos mecanismos mais utilizados para auxílio no diagnóstico e acompanhamento da evolução de portadores de autismo. Os resultados das avaliações realizadas em um determinado portador, podem ser usadas para criação de diversos tipos relatórios sobre a evolução do mesmo, facilitando dessa forma, a escolha de novas terapias que possam potencializar seus ganhos.

\section{Introdução}

O TEA (Transtorno do Espectro Autista) é relacionado a um conjunto de sintomas que comprometem a atividade cognitiva e de comunicação [Marinho and Merkle 2009]. Entre os sintomas que caracterizam o TEA, destacam-se atrasos na linguagem, dificuldade na comunicação e comportamentos estereotipados e repetitivos [Britto and Pizzolato 2016].

Para o diagnóstico e acompanhamento da evolução dos portadores de TEA, foram desenvolvidos questionários de rastreio que ajudam a identificar a evolução dos pacientes durante o período de tratamento. Entre os questionários desenvolvidos e validados, um dos dos mais utilizados é o CARS (Childhood Autism Rating Scale) [Pereira et al. 2007].

Durante o tratamento de portadores de TEA, a evolução do quadro clínico é mais valiosa e confiável quando baseados em múltiplas fontes de informação, incluindo observações do clínico, história do cuidador e demais terapeutas [Nascimento 2013]. 
Porém, a enorme quantidade de dados produzidos por vários profissionais de diversas áreas como psicologia, fonoaudiologia, psicopedagogia nem sempre é disponibilizada a todos os profissionais que acompanham a evolução do portador. Quanto maior a quantidade de informação disponível sobre um portador, mais eficientes serão as estratégias adotadas para conseguir ganhos significativos na evolução do seu quadro clínico.

Diante disso, o SATA (Sistema de Acompanhamento do Tratamento de Autismo) é uma ferramenta web para acompanhamento do tratamento dos portadores de TEA, disponibilizando aos profissionais resultados de avaliações realizadas sobre esses pacientes, fornecendo um histórico evolutivo ao longo do seu tratamento.

\section{Autismo}

O autismo é um transtorno caracterizado por prejuízos na interação social, atraso na aquisição da linguagem e comportamentos estereotipados e repetitivos, manifestando-se por toda a vida do portador. Segundo a Organização das Nações Unidas (ONU), cerca de 70 milhões de pessoas no mundo são acometidas pelo transtorno, sendo que, em crianças, é mais comum que o câncer, a Aids e o diabetes [Silva et al. 2012].

A primeira etapa do desenvolvimento infantil (entre 3 e 6 anos de idade), conhecida como primeira infância, é considerada primordial, pois é nessa fase da vida que a criança arquitetará uma base que o favorecerá por toda a existência [Silva et al. 2012]. O diagnóstico e a intervenção do autismo na primeira infância podem diminuir os déficits e comportamentos anormais associados ao transtorno, aumentando a qualidade de vida e independência funcional dos portadores.

A CARS (Childhood Autism Rating Scale) é um dos instrumentos mais utilizados na avaliação e acompanhamento da evolução do quadro clínico de crianças portadores de autismo e vem sendo traduzida, validada e utilizada nos mais importantes centros de diagnósticos e tratamento de autismo infantil. É um escala composta de 15 itens que auxiliam na identificação de crianças com autismo e as distingue de crianças com prejuízos no desenvolvimento sem autismo, tendo sua importância por conseguir diferenciar o autismo leve-moderado do autismo considerado grave ou severo [Pereira et al. 2007]. A ferramenta CARS é um facilitador do reconhecimento e classificação do autismo para clínicos e educadores, além de ser um instrumento claro para pesquisadores, uma vez que disponibiliza informações sobre o comportamento dos portadores assim como a gravidade dos sintomas.

\section{SATA (Sistema de Acompanhamento do Tratamento de Autismo)}

O SATA (Sistema de Acompanhamento do Tratamento de Autismo) é uma ferramenta web desenvolvida em PHP, Materialize e banco de dados Mysql. Ele tem como finalidade o acompanhamento do diagnóstico de portadores do autismo, servindo de base de dados permanente sobre o paciente e dando acesso aos profissionais envolvidos no seu tratamento a todas as avaliações realizadas em períodos anteriores.

Normalmente o portador de autismo é tratado por vários profissionais, como psicólogos, fonoaudiólogos, fisioterapeutas, psicopedagogos, professores e cuidadores. Porém, nem sempre as informações são compartilhadas entre esses profissionais para que possam ajudar de maneira eficiente no tratamento desse paciente. O SATA concentra esses 
VI Congresso Brasileiro de Informática na Educação (CBIE 2017)

Anais do XXVIII Simpósio Brasileiro de Informática na Educação (SBIE 2017)

dados para minimizar a dificuldade de compartilhamento das informações das avaliações, além de promover um mecanismo padronizado de avaliação a todos os profissionais envolvidos.

Para a avaliação dos pacientes, o SATA disponibiliza aos profissionais formulários para avaliações por meio da escala CARS, citada anteriormente, que fornece escores para 15 itens que auxiliam na identificação de crianças com autismo. Esses escores variam de 1 (dentro dos limites da normalidade) a 4 (sintomas autistas graves). A pontuação final varia de 15 a 60, e o ponto de corte para autismo é 30. No SATA, o resultado obtido na avaliação é comparado a valores pré-determinados pela técnica de rastreio ( 15 a $30=$ sem autismo, 30 a 36 = autismo leve/moderado, 36 a 60 = autismo grave) e indica o nível de autismo do portador ou até mesmo a inexistência do transtorno. Além do nível do autismo, o SATA emite relatórios de evolução em cada um dos 15 itens da CARS em períodos e grupos de profissionais selecionados pelo usuário do sistema, possibilitando assim a emissão de diversos relatórios ao neuropediatra para ajudar no diagnóstico e acompanhamento periódico e multiprofissional dos portadores do transtorno.

$\mathrm{Na}$ etapa de testes da aplicação, foram criados personagens fictícios, com base em portadores anônimos de TEA. Com esses personagens fictícios, foram realizadas simulações de funcionamento da aplicação. Os resultados mostraram a capacidade de avaliação e acompanhamento de acordo com os padrões estabelecidos pela técnica de rastreio utilizada.

\section{Conclusão}

O SATA é uma ferramenta desenvolvida para auxiliar no acompanhamento do tratamento de portadores de TEA. Ele é capaz de armazenar informações de diversas avaliações com base no questionário de rastreio de autismo CARS (Childhood Autism Rating Scale).

Conforme observado em simulações realizadas com personagens fictícios, as informações disponibilizadas no SATA podem ser relevantes durante o tratamento de portadores de TEA, pois possibilitam um melhor acompanhamento da situação do paciente por parte de diversos profissionais, entre eles, o neuropediatra.

\section{Referências}

Britto, T. C. P. and Pizzolato, E. B. (2016). Gaia: uma proposta de um guia de recomendações de acessibilidade de interfaces web com foco em aspectos do autismo. V Cngresso Brasileiro de Informática na Educação (CBIE 2016).

Marinho, E. A. R. and Merkle, V. L. B. (2009). Um olhar sobre o autismo e sua especificação. IX Congresso Nacional de Educação - EDUCERE, III Encontro Sul Brasileiro de Psicopedagogia.

Nascimento, M. I. . (2013). Manual diagnóstico e estatistico de transtornos mentais dsm-5. Artmed.

Pereira, A. M., Wagner, M. B., and dos Santos Riesgo, R. (2007). Autismo infantil tradução e validação da cars(childhood autism rating scale) para uso no brasil. Universidade Federal do Rio Grande do Sul.

Silva, A. B. B., Gaiato, M. B., and Reveles, L. T. (2012). Mundo Singular-Entenda o autismo. Fontanar. 